\title{
The Lazarus Effect: Healing Compromised Devices in the Internet of Small Things
}

\author{
Manuel Huber \\ Fraunhofer AISEC \\ Garching near Munich, Germany \\ mahuber@microsoft.com
}

\author{
Stefan Hristozov \\ Fraunhofer AISEC \\ Garching near Munich, Germany \\ stefan.hristozov@aisec.fraunhofer.de
}

\author{
Simon Ott \\ Fraunhofer AISEC \\ Garching near Munich, Germany \\ simon.ott@aisec.fraunhofer.de
}

\author{
Vasil Sarafov \\ Fraunhofer AISEC \\ Garching near Munich, Germany \\ vasil.sarafov@aisec.fraunhofer.de
}

\author{
Marcus Peinado \\ Microsoft Research \\ Redmond, United States \\ marcuspe@microsoft.com
}

\begin{abstract}
We live in a time when billions of IoT devices are being deployed and increasingly relied upon. This makes ensuring their availability and recoverability in case of a compromise a paramount goal. The large and rapidly growing number of deployed IoT devices make manual recovery impractical, especially if the devices are dispersed over a large area. Thus, there is a need for a reliable and scalable remote recovery mechanism that works even after attackers have taken full control over devices, possibly misusing them or trying to render them useless.

To tackle this problem, we present Lazarus, a system that enables the remote recovery of compromised IoT devices. With Lazarus, an IoT administrator can remotely control the code running on IoT devices unconditionally and within a guaranteed time bound. This makes recovery possible even in case of severe corruption of the devices' software stack. We impose only minimal hardware requirements, making Lazarus applicable even for low-end constrained off-the-shelf IoT devices. We isolate Lazarus's minimal recovery trusted computing base from untrusted software both in time and by using a trusted execution environment. The temporal isolation prevents secrets from being leaked through side-channels to untrusted software. Inside the trusted execution environment, we place minimal functionality that constrains untrusted software at runtime.

We implement Lazarus on an ARM Cortex-M33-based microcontroller in a full setup with an IoT hub, device provisioning and secure update functionality. Our prototype can recover compromised embedded OSs and bare-metal applications and prevents attackers from bricking devices, for example, through flash wear out. We show this at the example of FreeRTOS, which requires no modifications but only a single additional task. Our evaluation shows negligible runtime performance impact and moderate memory requirements.
\end{abstract}

Permission to make digital or hard copies of all or part of this work for personal or classroom use is granted without fee provided that copies are not made or distributed for profit or commercial advantage and that copies bear this notice and the full citation on the first page. Copyrights for components of this work owned by others than the author(s) must be honored. Abstracting with credit is permitted. To copy otherwise, or republish, to post on servers or to redistribute to lists, requires prior specific permission and/or a fee. Request permissions from permissions@acm.org.

ASIA CCS '20, October 5-9, 2020, Taipei, Taiwan

(c) 2020 Copyright held by the owner/author(s). Publication rights licensed to ACM. ACM ISBN 978-1-4503-6750-9/20/10 . \$ $\$ 15.00$

https://doi.org/10.1145/3320269.3384723

\section{CCS CONCEPTS}

-Security and privacy $\rightarrow$ Embedded systems security; Trusted computing.

\section{KEYWORDS}

trusted computing; cyber resilience; recovery; availability

\section{ACM Reference Format:}

Manuel Huber, Stefan Hristozov, Simon Ott, Vasil Sarafov, and Marcus Peinado. 2020. The Lazarus Effect: Healing Compromised Devices in the Internet of Small Things. In Proceedings of the 15th ACM Asia Conference on Computer and Communications Security (ASIA CCS '20), October 5-9, 2020, Taipei, Taiwan. ACM, New York, NY, USA, 14 pages. https://doi.org/10.1145/ 3320269.3384723

\section{INTRODUCTION}

With the Internet of Things (IoT) becoming increasingly pervasive and a focal topic in computing, more and more IoT devices are rolled out. Driven by cost savings and short product development cycles, a vast number of IoT business use cases have emerged, making IoT a disruptive technology. Examples are home automation [12], farming [42], sensor networks [37], Car2X [9], industrial IoT [48], or smart devices like tools [18], traffic lights [25] or vending machines [8], to name only a few. These devices are typically connected via (wireless) networks to a hub, a back-end server located in the cloud or managed by an enterprise. The growing importance of IoT deployments for public safety and business processes makes them an attractive target for attackers. This has been demonstrated by a large number of attacks $[10,29]$, such as the Mirai [30] or the Hajime botnet [44].

An important property of the IoT domain is that many devices with identical software stacks and configurations can likely be found in the field. This makes identified vulnerabilities or misconfigurations highly scalable, allowing attackers to potentially compromise a large number of devices. Especially small and cheap devices, oftentimes poorly secured, can be found in large numbers [17]. Even more alarming is the threat of attackers making such devices refuse communication and updates from the hub or permanently damaging them. In consequence, devices have to be manually recovered by replacing them or resetting them with clean software. As billions of devices (managed by a much smaller number 
of administrators) are deployed (often geographically dispersed), manual recovery becomes completely impractical.

As a solution to this problem, several cyber-resilient IoT architectures have been proposed $[5,52,54]$. These architectures enable remote recovery of infected IoT devices within a time bound regardless of compromise. State-of-the-art architectures [5, 52, 54] employ a minimal, early-boot recovery Trusted Computing Base (TCB) and a reset trigger that preempts compromised software. The recovery TCB ensures that only software authorized by the hub runs on the device. If no such software is present, the recovery TCB downloads the software from the hub and replaces the existing outdated or compromised stack. The reset trigger ensures that a reset into the recovery TCB will eventually happen even if software actively refuses the reset. Both the recovery TCB and reset trigger are isolated from untrusted software at runtime. This prevents malware from modifying the recovery TCB and from interfering with the reset trigger. An example of a reset trigger is the Authenticated Watchdog Timer (AWDT) [54]. After having been initialized, the AWDT will force a device reset after a certain time period, unless it is serviced with cryptographically protected "tickets". These tickets cannot be forged by software on the device but only be issued by the hub. As a consequence, as soon as the hub stops issuing tickets, e.g., when a zero-day vulnerability becomes known, or when the device behaves suspiciously, the AWDT will eventually time out and trigger a reset. A similar timer called "latchable WDT" [52] will power cycle a device within a specified time interval after its activation.

With resilient IoT architectures, we envision a world of selfhealing IoT deployments where all IoT devices can be reliably recovered even if they are compromised. However, existing cyberresilient IoT architectures which target primarily higher-end IoT devices do not address several critical real-world problems which must be solved to fulfill this vision even for low-cost microcontrollers. This includes

1) Hardware requirements. Current designs require hardware that typically does not exist or would incur additional cost on weaker, low-cost Commercial Off-The-Shelf (COTS) devices such as Microcontroller Units (MCUs). Examples are "storage latches" to write-protect the recovery TCB from untrusted software, or the AWDT peripheral that was realized previously as a separate MCU and attached to the main board [54]. For small MCU-based IoT devices, this makes the AWDT a feature as complex as the class of devices it is intended to protect. For storage latches, CIDER [54] relied on eMMC memory chips which are relatively complex and expensive and typically not available on small MCU-based devices.

2) Preventing attackers from disabling devices. Existing work neglects attacks in which the adversary tries to make a device unavailable. Examples include entering low-power or off states in which the reset trigger becomes inactive, wearing out flash memory [46], or disabling the networking hardware which is needed to replace corrupted software on the device.

3) Updates of the recovery TCB. After devices have been deployed, vulnerabilities in the recovery TCB itself may be discovered, or cryptographic requirements may change. It is essential to patch such vulnerabilities in a timely manner before they can be exploited by attackers.
This paper presents the Lazarus system, a cyber-resilient IoT architecture that solves these three problems. Lazarus targets lowcost COTS MCUs. We take advantage of the recent addition of a general-purpose Trusted Execution Environment (TEE) to low-end ARM MCUs in the ARMv8-M architecture. As the ARMv8-M replaces earlier models, we expect TEEs to become widely available in low-end MCUs in the coming years. A key observation in the design of Lazarus is that the security hardware required by cyber-resilient architectures can be emulated by software running inside a TEE. Furthermore, the TEE can mediate access by untrusted software to critical system state.

In particular, we implement storage write protection latches and the AWDT in software running in the TEE. We call our reset trigger "TEETrigger." With this design, we enable the AWDT to be realized on existing COTS devices and do not make assumptions on trusted peripherals for storage latches and reset triggers. Our design prevents attackers from making devices permanently unavailable by interposing between untrusted software and peripherals that are critical for availability. We call these "critical peripherals" and use the TEE to regulate or block access by untrusted software, preventing their misuse. Finally, existing IoT architectures use the Device Identifier Composition Engine (DICE) for authentication and attestation to verify whether authorized software is present on the device. This enables the hub to authenticate the deployed software stack. However, as the hash of the boot code is part of the DICE attestation identity, an update of the recovery TCB will give the device a new DICE identity that the hub cannot predict. This makes it impossible for the hub to recognize device identities after a TCB update and to verify if an update was actually installed or not. To overcome this problem, we propose an extension to DICE, which we call DICE++. Our extension enables devices to provide proof that a designated update was applied and sustains a device's identity across updates of the recovery TCB.

We demonstrate the effectiveness of Lazarus on low-end devices by implementing a prototype on an existing COTS low-end MCU based on the new ARMv8-M processor family. We make use of the isolation capabilities of TrustZone-M to isolate the recovery TCB from untrusted software and to build an AWDT without requiring additional hardware. We make the following contributions:

- We design Lazarus, a resilient IoT architecture for off-theshelf IoT devices. The design of Lazarus respects even the weakest class of IoT devices and protects against attackers actively aiming to render devices useless. With TEETrigger, Lazarus includes a zero-cost reset trigger, the first of its kind. Our design relies on a TEE which is provided by modern processors.

- We design DICE++ which enables updates of the recovery TCB without loss of device identity.

- We implement a prototype on Lazarus on a recent ARM Cortex-M33 based MCU, the NXP LPC5500 [33]. We leverage the new TrustZone-M extension for ARM Cortex-M CPUs and ensure portability across different ARMv8-M devices. Our implementation includes a full setup featuring an IoT hub, device provisioning and update functionality.

- We demonstrate how to protect an embedded OS, FreeRTOS [39], with our prototype. The OS modifications are limited 
to adding a single supplementary task for extending TEETrigger in coordination with the hub.

- We provide a security discussion and an evaluation of the runtime and boot time performance impact as well as of the memory requirements, showing the practicability of Lazarus.

\section{BACKGROUND}

This section provides background for the rest of the paper.

\subsection{Latches}

The code and data of Lazarus on storage requires protection while untrusted software executes. Latches [54] can be used to protect critical code and data from being overwritten or read out by untrusted software. Conceptually, a latch is a state machine with two states, open and locked. A reset puts the latch into the open state. Software can put the latch into the locked state (e.g., by writing to a hardware register). Importantly, the only action or event that can return the latch to the open state is a reset. A latch has an associated security function which allows some action (e.g., writing to certain flash regions) if and only if the latch is in the open state.

Latches allow trusted boot software that runs directly after a reset to have full access to all hardware resources but to selectively disable access to some of these resources to (less trusted) software running subsequently on the device. A read-write latch (RWLatch) can be used to protect secrets, for instance, and a write latch (WRLatch) to protect the integrity of data.

\subsection{DICE}

DICE $[49,50]$ is an industry standard designed to enable attestation on low-end devices with only minimal hardware support. DICE has been adopted by major MCU manufacturers such as NXP [34] or Microchip [26] and cloud providers [38]. DICE is significantly more light-weight than alternatives such as the TPM [45]. A DICE device has a unique secret, the Unique Device Secret (UDS) that is protected by a latch. After a reset, DICE measures the first mutable software component $\mathcal{M}$ (e.g., a boot loader) and uses a one-way function to derive a symmetric key, called Compound Device Identifier (CDI), from this measurement and the UDS. Next, DICE makes the UDS inaccessible until the next reset, discloses the CDI to $\mathcal{M}$ and invokes $\mathcal{M}$. This provides $\mathcal{M}$ with an identity that is unique to both $\mathcal{M}$ and the device and that forms the foundation for DICE attestation.

Critically, the DICE standard prescribes that the computation of the CDI must not be performed by mutable software, i.e., by software that can be modified or updated. While a device manufacturer may choose to implement the computation of the CDI in software, this software is fixed (e.g., burned into ROM) and not under the control of the device owner. This fact together with the method by which the CDI is derived implies that (a) any modification of $\mathcal{M}$ will result in a different CDI and (b) the new CDI is unpredictable, as the UDS is only known to the device's DICE component, and the CDI is derived from the UDS via a one-way function.

DICE can be leveraged for lightweight device attestation, for instance for IoT devices connecting to a hub [51]. $\mathcal{M}$ (or code $\mathcal{M}$ trusts) uses the CDI to derive two asymmetric key pairs, called the DeviceID and the AliasID, and uses the DeviceID private key to sign the AliasID certificate. Then, $\mathcal{M}$ removes all remnants of the
CDI and the DeviceID private key and provides untrusted software only with the AliasID key pair and the certificates. This results in a cryptographic chain from the UDS and the CDI to the DeviceID and the AliasID based on the state of a device's software stack. Untrusted software can use the AliasID as a device identifier for authentication and attestation.

\subsection{MCU Model}

Microcontroller units typically combine one or two low-end microprocessor cores with moderate amounts of RAM and flash memory and various simple devices such as watchdog timers and security features such as DICE. With the introduction of ARMv8-M and TrustZone for Cortex-M, TEEs are rapidly becoming prevalent even in the low-end MCU market.

This paper is based on an MCU model whose processor(s) support two privilege levels (privileged and unprivileged) which are comparable to user and kernel mode and which allow a simple "operating system kernel" to protect itself from applications. In addition, our MCU model supports DICE and, more importantly, a TEE. The assumed DICE support is not critical because, as we will show, Lazarus can implement DICE in software.

Our model of a TEE is based on TrustZone for Cortex-M, the de facto standard TEE for MCUs. Our TEE model isolates between two operating environments: a higher-privileged trusted world and a lower-privileged normal world. After a reset, boot code can assign various resources to either the trusted or the normal world. This includes devices as well as RAM and flash which can be assigned to either the trusted or the normal world in chunks of different granularities. Code running in the normal world can only access those resources that have been assigned to it.

\section{THREAT MODEL}

The software on the device is composed of Lazarus and the main IoT application logic. The latter may include an OS and applications running on it, a hypervisor, or bare-metal applications and is subject to compromise by a remote attacker. We assume the attacker to be capable of arbitrarily compromising this untrusted software. This includes the possibility that attacker code persists across device resets. An example is the exploitation of software vulnerabilities or misconfigurations of the OS or application logic. With these capabilities, the attacker is able to control common IoT devices at will and to make them unavailable.

Lazarus is composed of its core TCB and a downloader, i.e. a networking stack that allows Lazarus to communicate directly with the hub. We assume the core TCB to be immune to compromise as it is small and well isolated from a potential attacker. In contrast, we only assume the more exposed downloader to work correctly when not under attack. The core TCB puts protections in place that allow easy recovery from a compromise of the downloader.

We assume the hardware to work correctly according to specification. For instance, the attacker cannot alter code in ROM, or interfere with peripherals shielded by the TEE. However, the attacker may be able to leak secrets from the TEE, e.g., as demonstrated by Lapid and Wool [24]. The attacker is unable to efficiently break state-of-the-art cryptographic primitives. 
The attacker may eavesdrop on the communication channel between device and hub as well as forge or tamper with messages. However, the attacker can block the channel only for a limited amount of time. We consider prolonged attacks on the communication channel to be detectable and remediatable by network operators.

The focus of this paper is the protection of IoT devices. The hub, a web service likely to reside in a commercial cloud, can be protected with the full array of industrial-strength IT security solutions and is outside scope of this paper. Thus, we consider the hub to be immune to attacks. The same holds for the environment in which devices are provisioned. As we focus on scalable attacks, we leave physical attacks requiring proximity of the attacker to a device out of scope.

\section{DESIGN OF LAZARUS}

In this section, we describe the design of Lazarus, for which we combine several hardware and software building blocks. To overcome the three problems of existing cyber-resilient IoT architectures listed in the introduction, we set the following design goals:

DG-I Strong isolation of Lazarus from untrusted software while minimizing hardware requirements. This makes Lazarus applicable on a wide range of low-cost MCU-based IoT devices.

DG-II Prevent malware from damaging devices or making them permanently unavailable.

DG-III Provide a stable device identity and attestation of the software stack even in case of an update to Lazarus.

DG-IV Lazarus should require only minimal changes in existing OS and application software.

In support of DG-I, we will realize the reset trigger TEETrigger and latches in software. We achieve DG-II by regulating access by untrusted software to peripherals critical for the availability of devices such as a Flash Memory Controller (FMC). Lazarus requires only the following hardware:

Entropy source to ensure freshness and non-forgeability of messages Lazarus exchanges with the hub, e.g., a Hardware Random Number Generator (HRNG).

Orderly reset to allow Lazarus to execute deterministically from a clean state after reset, regardless of prior state.

TEE for realizing latches, TEETrigger, and for controlling access to critical peripherals.

Ordinary watchdog timer (WDT) as a building block for TEETrigger. This simple device is commonly included in MCUs to detect software crashes and reset the device. Software is expected to service the WDT periodically. The WDT will reset the device if it has not been serviced for some amount of time.

These hardware features exist on a broad set of modern MCUs. In contrast to CIDER [54], Lazarus requires neither hardware latches, nor an external reset trigger.

Lazarus is split between a trusted boot loader and a runtime component that executes inside the TEE concurrently with the untrusted software which runs outside the TEE. After a reset, the boot loader runs DICE++. Subsequently, similar to the CIDER boot loader, it may download and install updates to the untrusted software. Finally, it initializes the Lazarus runtime component in the TEE and transfers control to the untrusted software.

We first describe how we construct latches and TEETrigger to isolate Lazarus from untrusted software (DG-I). Then, we focus on the isolation of critical peripherals (DG-II) and on our extension of DICE (DG-III). Finally, we describe how we use these mechanisms to design our end-to-end system and refer to DG-IV.

\subsection{Construction of Latches}

Lazarus has to protect its binaries and data in flash from being corrupted by the untrusted software. We configure the TEE to make the flash range that stores Lazarus code or data inaccessible to the untrusted world. Effectively, this constitutes a latch, as the restrictions will stay in place until the next reset, and the untrusted software has no means of unlocking the latch. We use the same mechanism to protect RAM for the Lazarus runtime component running in in the TEE.

DICE without hardware support: On MCUs without hardware support for DICE, we can use our TEE-based latches to build DICE in software. The software consists of the UDS (i.e., a unique secret) and code that hashes the next binary and derives the CDI from it and the UDS. This code runs immediately after a reset. Its last action is to read-write-latch itself including the UDS, thus making itself and the UDS inaccessible until the next reset. Control and the CDI are then transferred to the Lazarus boot code.

\subsection{Construction of TEETrigger}

We use the TEE and the WDT to construct our reset trigger TEETrigger. TEETrigger is software running in the secure world which exposes the standard AWDT interfaces [54] to the normal world.

A simple TEETrigger version could implement the AWDT initialization call by storing the hub's public key (provided as a parameter) and starting the hardware WDT with the timeout value from the AWDT initialization call. A second AWDT function generates a nonce using the HRNG, stores it in trusted memory and also returns the nonce to the untrusted caller. Untrusted application code can use the nonce to request a deferral ticket for the AWDT from the hub. If the hub issues such a ticket, the untrusted application code can use the third AWDT interface to request deferral of AWDT expiration and, thus, device reset to be postponed. TEETrigger could implement this third call by using the public key that was provided during initialization to verify the signature on the deferral ticket and only servicing the WDT if the signature verification succeeds and the nonce from the deferral ticket matches the stored nonce.

In practice, the situation is complicated by a mismatch between the timeout intervals supported by most existing hardware WDTs (at most few minutes) and those expected of AWDTs (hours to weeks). In order to support AWDT timeout intervals that are not limited by those of the WDT hardware, we take advantage of a WDT feature that causes the WDT to issue an interrupt a short time before resetting the device. This interrupt warns software that a device reset is imminent. We maintain a counter which is set to a positive value during AWDT initialization. Our interrupt handler will decrement the counter and service the WDT if and only if the result is not negative. For example, if the WDT hardware only 


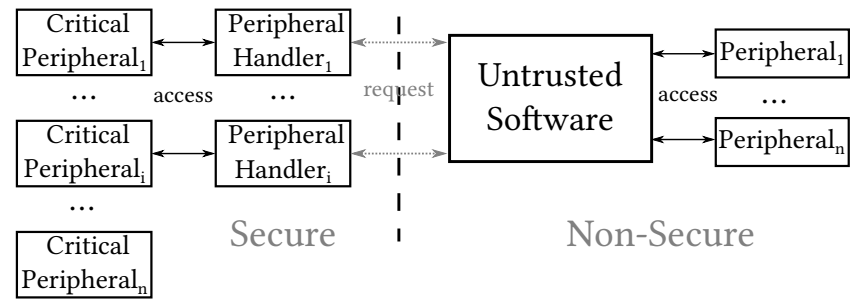

Figure 1: Interposition between untrusted software and critical peripherals through handlers in the TEE.

supports timeout intervals of up to one minute, and we desire a 10 minute timeout for the AWDT, we can set the counter to 10 during initialization or after a valid deferral ticket has been received. This will cause TEETrigger to service the hardware WDT ten times for a ten minute timeout interval.

\subsection{Isolation of Critical Peripherals}

Untrusted software should be able to execute unconstrained and thus be allowed to access peripherals. However, to assure recoverability, we must regulate its access to certain critical peripherals. Otherwise, untrusted code could, for instance, put devices into an irrecoverable low-power state, wear out flash, or permanently disable peripherals used for communication. This would make devices permanently unavailable and could even cause physical damage. Therefore, the Lazarus runtime component running in the trusted world of the TEE interposes between the untrusted code and these critical peripherals. We define a set of entry points into the TEE to allow untrusted software controlled access to critical peripherals. Figure 1 depicts this with "peripheral handlers" serving as entry points into the TEE.

An example of illicit use of critical peripherals is excessive flash writes by untrusted software. Even though the storage of trusted components of Lazarus may be latched using the TEE, excessive writes to unprotected storage locations could still cause flash to wear out. flash writes are handled via an FMC. This is why we define the FMC as a critical peripheral to be only accessible from within the TEE. For persisting data, untrusted software must then use our entry point to the TEE, where our trusted FMC handler manages write requests. Note that writing to flash is generally a slow operation. Interposing on such operations will have a limited performance impact.

Untrusted software might receive data from the hub, such as updates, or retrieve other data (e.g., from sensors) it has to store. When our flash write handler in the TEE receives a flash write attempt, it only allows:

- Writing to unprotected flash memory areas (areas that are not latched). This includes areas storing code and data of untrusted software and a staging area for storing updates. Writes to latched areas (storing Lazarus) are prohibited. This prevents untrusted software from circumventing the TEE's WRLatch for Lazarus code by using the handler.

- A reasonable amount of flash writes. We assume that for flash write events, a reasonable threshold or rate limitation

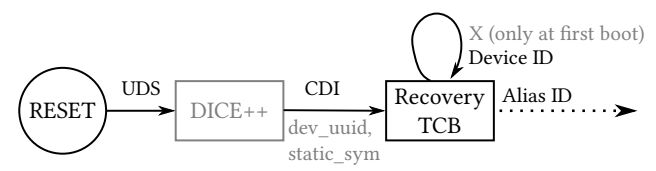

Figure 2: Modification to DICE (in gray) to exchange a shared secret and static identifier with the hub at first device boot.

to regulate flash writes can be determined at design time of an IoT use case.

The FMC is one example of a critical peripheral and its presence or criticality may depend on the IoT use case and device type. The absolute minimum set of critical peripherals that need to be protected for Lazarus are:

- Storage controllers allowing to write persistent storage subject to wear out.

- Power control peripherals that can put the device into low power modes or turn the device off.

- The WDT used by TEETrigger.

If a handler in the TEE detects illicit use of peripherals, it will reset the device. This allows remediation through Lazarus and can help the hub detect faulty behavior or compromise of business logic. Other peripherals such as networking devices or other storage controllers may be critical depending on the device type and IoT use case. Our prototype demonstrates that adapting the untrusted software to this protection mechanism requires only minor adaptations to certain library function calls (DG-IV).

\subsection{Extension of DICE}

To achieve design goal DG-III, we introduce a mechanism that allows updating early-boot code like Lazarus without loss of device identity. Updating early boot code like Lazarus is essential in practice, e.g. when updating cryptographic implementations or when an exploitable vulnerability has been identified. However, updating an early-boot component like Lazarus results in an unpredictable CDI and thus in a new DeviceID and AliasID. A hub knowing devices and their identities has no means of relating the new and old DeviceID after an update and has no way to verify that the update was indeed applied.

As a practical solution for updating early-boot code without loosing the device identity, we propose DICE++ as an extension to DICE. DICE++ enables devices to provide the hub with cryptographic proof of identity after a change in the DeviceID. This allows the hub to associate the old and the new DeviceID and AliasID and to verify that devices have properly applied their early-boot code update. DICE++ thus minimizes the amount of code that cannot be updated in practice. Our design of DICE++ can supplement existing DICE implementations on shipped devices, which we demonstrate in Section 5. The first part of this section describes device provisioning while the second part explains device authentication after an update of early-boot code.

Device Provisioning. Our idea for re-associating device identities after an update of an early-boot component is to use a static secret and identifier shared between device and hub. We create the secret and identifier on the device in a secure environment during 


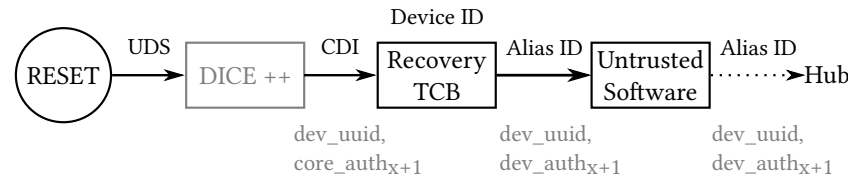

Figure 3: Modification to DICE key derivation and identitybased attestation (in gray) for updating the recovery TCB.

provisioning and exchange it with the hub. After an update, the device with a new DeviceID uses the static identifier for making an "identity claim". The hub requests proof of possession of the respective shared secret to verify a device's identity claim.

At the first boot of a device, DICE++ randomly generates the static identifier dev_uuid. Further DICE++ derives the shared secret static_sym as follows: static_sym :=KDF(UDS,dev_uuid). The secret static_sym is derived with a one way function based on the UDS and the static identifier, allowing no conclusions on the UDS. Both dev_uuid and static_sym remain unchanged when an early-boot component is updated. We use a secret different than the UDS to share with the hub, because static_sym is less security critical. static_sym is only used for re-association, not for regular attestation/authentication. Device identities are not compromised should static_sym ever leak. In contrast, if the burnt-in UDS leaks, the device is irrecoverably compromised. In such cases, we can regenerate dev_UUID, which causes derivation of a new static_sym, avoiding loss of a device.

Being in a secure environment, dev_uuid, static_sym and the initial DeviceID are read out and transferred to the hub. For this purpose, DICE++ provides dev_uuid and static_sym once at first boot to the next layer. At this time, early-boot code and environment can still be trusted. It is also possible to exchange dev_uuid and static_sym online after enrollment. In this case, the early-boot component generates the structure $X$ at initial boot and sends it to the hub on first connection:

$$
X:=\operatorname{sig}\left(e n c\left(d e v \_u u i d \mid s t a t i c \_s y m\right)^{H u b_{p u b}}\right)^{\text {DeviceID }}{ }_{\text {priv }}^{1}
$$

This means that the early-boot component encrypts static_sym and dev_uuid with the hub public key and signs it with its initial DeviceID private key, enabling confidential and authenticated transmission. The derivations at first boot are depicted in Figure 2.

Re-Association after Update. At every boot, DICE++ generates an additional key other than the CDI for the early-boot component. We call this key core_auth, which DICE++ derives as follows:

$$
\text { core_auth }:=K D F\left(\text { static_sym, hash }\left(M_{x} \mid \text { dev_uuid }\right)\right)
$$

This key depends on the current version $x$ of the early-boot component $\mathcal{M}$ on the device and allows it to authenticate itself. DICE++ hashes the early-boot component appended with the identifier dev_uuid and uses it with static_sym as input to a Key Derivation Function (KDF). The key core_auth $h_{x}$ can only be computed with knowledge of static_sym and indicates that the software stack $\mathcal{M}$ in version $x$ was measured on the device with dev_uuid. An update to version $x+1$ causes an independent derivation of the key core_auth $h_{x+1}$. Only DICE++ and the hub are able to derive this key as it depends on static_sym and thus cannot be forged or predicted.

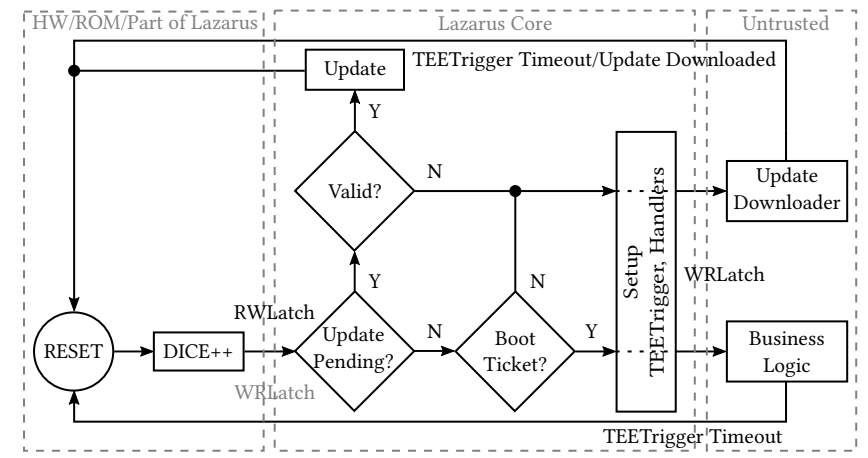

Figure 4: Boot flow of Lazarus including latching, TEETrigger initialization and peripheral handler setup.

The early-boot component receives this key from DICE++, which it uses to derive an "identity token", which we call dev_auth. Using dev_aut $h_{x+1}$, a modified early-boot component can prove to the hub that it was successfully updated and is running on exactly the device with the prior DeviceID. The derivations for core_auth and dev_auth are illustrated by Figure 3. To compute dev_aut $h_{x+1}$, the early-boot component uses core_aut $h_{x+1}$ as key for a Hash-based MAC (HMAC) over the new DeviceID and dev_uuid, i.e.,

$$
\text { dev_auth } h_{x+1}:=H M A C\left(\text { core_aut } h_{x+1}, \text { DeviceID } D_{p u b}^{x+1} \mid \text { dev_uuid }\right)
$$

dev_aut $h_{x+1}$ can only be computed by an early-boot component exactly in version $x+1$ running on the device dev_uuid and belongs to the DeviceID in version $x+1$. dev_auth $h_{x+1}$ depends on core_auth $h_{x+1}$ which is only present after a correct update to version $x+1$. Thus, dev_auth $h_{x+1}$ cannot be forged either. Early-boot code can now pass dev_aut $h_{x+1}$ and dev_uuid to the next layer to handle the identity claim, for instance by integrating it into the DeviceID certificate. When the hub receives a claim of a device with DeviceID in version $x+1$ to be identified as device with dev_uuid, the hub requests the identity token. The hub computes the expected identity token on its side as all parameters are known to the hub. In case the received and expected token match, re-association is complete and the new DeviceID accepted.

\subsection{End-to-End System}

This part describes how we compose our TEE-based latching, our TEETrigger, the protection mechanism for critical peripherals and DICE++ in our end-to-end system, Lazarus. We use CIDER [54] as a basis for this. Figure 4 illustrates the boot flow of Lazarus. Our first step is to apply temporal isolation, i.e., to execute the core functionalities of Lazarus before untrusted software like update download functionality or business logic executes. This means that the core part of Lazarus executes directly after an orderly reset.

After a reset, we execute DICE++ (including DICE, run by the $\mathrm{MCU})$. After activating the latches, Lazarus executes its main functionality "Lazarus Core" on the next lower security tier in the TEE.

Lazarus Core checks whether an update for business logic or for Lazarus itself is pending, i.e., whether an update candidate was stored in the "staging area" on persistent storage. If so, Lazarus Core verifies the integrity and authenticity of the update using a public 
key it received during device provisioning and a version number. If the update is valid, Lazarus Core applies the updates and resets. Otherwise, e.g., if it has been tampered with in the communication channel, Lazarus Core executes its "update downloader". The downloader contacts the hub to retrieve authenticated software.

The downloader, technically part of Lazarus, has a significant attack surface, as it has to handle arbitrary packets from the internet. Lazarus Core thus considers it untrusted software. This is why Lazarus executes the update downloader in the same security tier as business logic outside the TEE. Before executing untrusted software, Lazarus Core WRLatches itself including the downloader on storage. This gives the update downloader the same storage protection as Lazarus Core. In addition, Lazarus Core WRLatches the main memory region occupied by trusted runtime components running alongside untrusted software. Further, Lazarus Core initializes TEETrigger and constrains access to critical peripherals. Lazarus Core also derives the respective DeviceID and AliasID key material according to the DICE specification for untrusted software.

In case no update is pending, Lazarus Core checks for the presence of a "boot ticket" in the staging area. The boot ticket is an accreditation of the hub to boot one time into business logic without requiring further interaction with the hub through the update downloader. Without a valid boot ticket present, Lazarus Core tries to acquire a boot ticket using the update downloader. The downloader attests the device's software stack to the hub by presenting the DeviceID and AliasID. If the hub is satisfied, it issues a boot ticket. Like a deferral ticket, the boot ticket is a hub-signed data structure that contains a nonce for ensuring freshness. Lazarus Core generates and stores a fresh nonce at each reset. Upon reset, Lazarus Core can verify the signature of a staged boot ticket and compare the contained nonce with the stored, old nonce.

If a valid boot ticket is present, Lazarus Core boots into the business logic. This stack may execute as long as it can acquire fresh deferral tickets from the hub. Business logic can also authenticate itself to the hub using its AliasID and DeviceID credentials and may retrieve boot tickets. Ticket acquisition from the hub can be implemented as a task in an OS.

The only parts of Lazarus active while untrusted software runs are TEETrigger and the logic regulating access to critical peripherals. This code has no access to secrets, making side channel attacks pointless. The TEE exposes a small set of well-defined interfaces to allow untrusted software to interact with TEETrigger and to use critical peripherals.

Similar to [54], detection of compromise and identification of vulnerabilities is not in our scope. We consider such lines of work orthogonal to our approach [53]. Availability of devices due to attacks on the communication channel or by making the hub unavailable are discussed in [54].

\section{IMPLEMENTATION}

Our goal is to demonstrate that Lazarus can be implemented even on low-cost devices. Therefore, we implemented a prototype for a low-end COTS MCU from the NXP LPC5500 series [33]. The price for MCUs in this category ranges from cents to a few dollars [17]. While first MCUs based on the ARMv8-M specification have only

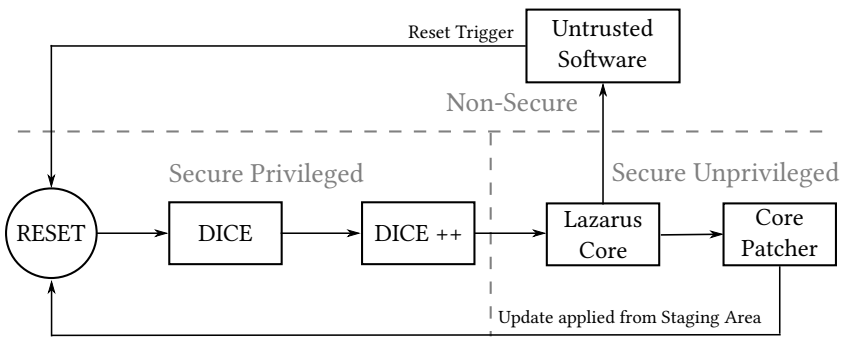

Figure 5: Overview of the implemented functionality along with execution modes and privilege levels.

recently been released, we expect this generation of MCUs to find wide adoption on the market.

We chose the LPCXpresso55S69 development board [32] as a target for our implementation. The board is equipped with an NXP LPC55S69 (revision 0A) MCU, peripherals such as an accelerometer and several expansion ports. The LPC55S69 MCU features a dual core 32-bit ARM Cortex-M33 processor based on the ARMv8-M architecture [3] running at $96 \mathrm{MHz}$ with TrustZone-M, $320 \mathrm{~KB}$ of SRAM, $640 \mathrm{~KB}$ of flash, a watchdog timer, a HRNG, crypto acceleration and DICE support [31,35]. Since our board does not include networking hardware, we attached an off-the-shelf Wi-Fi chip, the ESP8266 [13], to one of the board's USARTs via one of the expansion ports. The ESP8266's serial port connection to the USART runs at 115,200 baud, thus limiting the network bandwidth to about 14 $\mathrm{KB}$ per second.

With TrustZone-M, the Cortex-M CPU can be in secure or nonsecure mode. Each mode has a privileged and an unprivileged level. Non-secure execution can invoke the secure world through NonSecure Callable (NSC) functions. Peripherals can be configured as fully secure, fully non-secure or split into secure and non-secure partitions. When the CPU is in a secure state, it can access both the secure and the non-secure world. In contrast, the processor can access only the non-secure resources when it is in a non-secure state.

The MCU hosts a secure AMBA AHB5 controller [2, 35] which allows configuring the whole memory map including flash, RAM and peripherals as secure/non-secure and privileged/unprivileged. The ARM AMBA AHB5 protocol introduces signaling for secure and non-secure transactions and therefore extends the TrustZone technology from the processor to the entire system. Lazarus can be implemented on any ARMv8-M microcontroller that allows securing our critical peripherals, which holds for every ARMv8-M microcontroller currently on the market.

Our prototype splits the different functions of Lazarus into separate binaries running in different tiers as illustrated in Figure 5 and explained in the following. The deployment of the binaries and their execution in secure and non-secure mode led to the flash layout depicted in Figure 6.

We implemented the Lazarus prototype in C. Table 1 summarizes the Lines of Code (LoC) of our prototype, measured with Lizard [47]. The Lazarus functionality executed in the secure world, consisting of DICE++, Lazarus Core (LZ Core) and Core Patcher $(C P)$, has less than $5 \mathrm{k} \mathrm{LoC}$ in total. The update downloader $(U D)$ has about $1.4 \mathrm{k}$ 
Table 1: LoC of the different components of our prototype.

\begin{tabular}{ccccccc}
\hline DICE++ & LZ Core & $C P$ & $U D$ & Crypto & CMSIS & NXP \\
\hline 1,668 & 2,753 & 571 & 1,359 & 3,564 & 1,764 & 5,682 \\
\hline
\end{tabular}

LoC. In addition, our prototype uses parts of the RIoT crypto library [27], and parts of the CMSIS and NXP hardware-specific code.

Both CMSIS and NXP are relatively large bodies of headers and support libraries that implement a hardware abstraction layer for a large number of different hardware features of the Memory Protection Unit (MPU). Our prototype uses only a small number of these hardware features and a similarly small fraction of the CMSIS and NXP code. We excluded code (e.g., header and C files) that our prototype obviously does not use from the counts in Table 1 . Even so, the CMSIS and NXP numbers still overestimate the amount of code used in our prototype, as separating all unused code would have been a complex task.

DICE and DICE++. As the NXP LPC55S69 supports DICE according to the Trusted Computing Group (TCG) specification [50], we do not have to implement DICE ourselves. DICE executes directly out of ROM after device reset before any other software is executed. For this reason, we cannot modify DICE, which is why we implemented DICE++ as a separate binary executed right after DICE. The MCU executes the first code from flash after DICE from address $0 \times 00000000$, which is where we placed DICE++. We calculate static_sym using HMAC-SHA256 at each boot. Instead of using the UDS (which we cannot access because DICE latches it), we derive static_sym from the CDI which DICE passes to DICE++. This does not weaken the security model because we remove the CDI before passing control to the next layer and hand over only a re-derivation of the CDI:

$$
C D I^{\prime}:=H M A C\left(C D I, H a s h\left(L a z a r u s \_C o r e\right)\right)
$$

DICE++ derives core_auth and CDI' using HMAC-SHA256.

For DICE++, we only need to protect dev_uuid and the DICE++ binary itself from being overwritten by the following layers. To ensure this, we execute DICE++ in secure privileged mode and all following layers as either secure unprivileged or non-secure. We also use the secure AHB controller to WRLatch DICE++ including dev_UUID. The secure AHB controller can only be configured when executing secure privileged. Once activated, it cannot be reconfigured or switched off until the next reset. This forces us to activate all required latches at once during TEE configuration. For this reason, we configured the secure AHB controller in DICE++ to also WRLatch code and data of Lazarus core that we execute in secure unprivileged mode. We also use the secure AHB controller to map the critical peripherals into the secure world before executing untrusted software.

Lazarus Core. Lazarus Core is responsible for deriving the token dev_auth for Lazarus updates, for DeviceID and AliasID derivation, for boot ticket verification, for boot nonce generation, for checking if authorized code is present or whether an update must be applied, for verifying and applying staged updates, and for regulating access to critical peripherals.

\begin{tabular}{|c|c|c|c|c|c|c|c|}
\hline \multicolumn{3}{|c|}{ Secure Flash } & \multicolumn{5}{|c|}{ Non-Secure Flash } \\
\hline DICE++ & $\begin{array}{c}\text { Lazarus } \\
\text { Core }\end{array}$ & $\begin{array}{c}\text { Core } \\
\text { Patcher }\end{array}$ & $\begin{array}{c}\text { Update } \\
:\end{array}$ & $\begin{array}{c}\text { Business } \\
\text { Logic }\end{array}$ & Data & $\begin{array}{l}\text { Staging } \\
\text { Area }\end{array}$ & \\
\hline
\end{tabular}

Figure 6: Layout of the binaries in flash memory along with their execution mode.

For secure communication with the hub, Lazarus Core derives the DeviceID and AliasID from CDI' and provides the signed AliasID to untrusted software as input mapped to specific RAM regions. For these derivations, Lazarus uses elliptic-curve cryptography with the prime256v1 curve. The public keys are embedded into X.509 certificates and the DeviceID certificate is signed by the hub in order to establish a Public Key Infrastructure (PKI). For the derivation of dev_auth, Lazarus Core uses HMAC-SHA256.

To regulate access to critical peripherals and to interact with TEETrigger, Lazarus Core sets up NSC functions as an interface for untrusted software. We implemented several of these NSC functions, i.e., handlers, for controlling access to critical peripherals. For our implementation we considered the FMC, the power control peripheral, and the WDT as critical [35]. The handler for the WDT implements the TEETrigger deferral logic. For deferral ticket nonce generation, the handler uses the HRNG.

Our handler for the power control peripheral ensures a device can only be put into those low-power states that do not affect TEETrigger. Its underlying WDT is driven by the MCU's FRO_1MHz clock. Whether this clock is running depends on the power state of the MCU. In active and sleep mode, it is enabled. However, in deep sleep, the state of the clock can be configured off by software. In the power-down modes the clock is off, which is why we block deep sleep and power-down requests.

Our handler for the FMC allows untrusted software only writes to flash memory allocated to untrusted software and to the staging area. Untrusted software thus remains capable of persisting data to specific flash regions, e.g., for providing boot and deferral tickets or staging updates. In addition, the handler implements a simple threshold scheme that disallows excessive flash memory writes. The protection of critical peripherals is not transparent to untrusted software. For this purpose, we modified the relevant libraries to use the handler function instead of directly interacting with the peripheral.

Core Patcher. In general, Lazarus Core is responsible for verifying and applying updates. However, as it cannot update itself in flash, in case a Lazarus Core update is present on the staging area, Lazarus Core only verifies this update and then invokes the Core Patcher. The Core Patcher's sole responsibility is to apply the Lazarus Core update. Like Lazarus Core, the Core Patcher runs in the secure world and can only be invoked by Lazarus Core itself. This design makes it possible to update all binaries except DICE++.

Update Downloader. The update downloader is part of Lazarus, but as it implements a networking stack for communicating with the hub, it is considered untrusted software. This is why we execute it in the non-secure world. Our handler for the FMC peripheral 
ensures that the update downloader can only write to specific areas in flash memory and thus not overwrite itself or other parts of Lazarus in case of compromise.

We used the ESP8266 Wi-Fi chip [13] for TCP/IP communication with the hub and implemented our own format for headers for binary updates. We sign all messages exchanged between the device and the hub with their respective private keys and ensure the freshness of critical messages using nonces for tickets and version numbers for updates. We implemented the update downloader as a bare-metal application. An advantage of our implementation is that the embedded OS Lazarus protects does not necessarily need to implement its own update mechanism.

Our prototype treats the ESP8266 as a fixed-function peripheral that is not subject to compromise. However, the ESP8266 has been compromised in the past [7]. The ESP8266 is effectively another small microcontroller running its own firmware stored in flash. It also exposes a firmware update interface. We disabled this interface by connecting its GPIO0 pin to $3.3 \mathrm{~V}$.

Vulnerabilities in the ESP8266 firmware might still allow an attacker to overwrite it and thus completely cut off our network connection. Such attacks might originate with the untrusted software or on the network. One can envision several defenses based on Lazarus or CIDER primitives to protect the ESP8266. For example, a more robust version of the ESP8266 could write-latch its firmware early in boot, such that it cannot be overwritten even if a potential vulnerability is exploited. Lazarus could also support ESP8266 firmware updates and treat the ESP8266 as a critical device by placing trusted handlers between the ESP8266 and untrusted software.

Business Logic. We implemented two applications representing real-world use cases. The first application is a traffic light controller which controls LEDs via GPIO. The second application periodically measures the temperature through an ADC sensor and records the values in flash. Both applications run as tasks on FreeRTOS We implemented the functionality for acquiring boot and deferral tickets as a separate task in FreeRTOS. This task uses the ESP8266 for the communication with the hub.

$H u b$ and Provisioning Environment. Neither the hub nor the provisioning environment are part of the core Lazarus device implementation. However, in order to be able to perform a complete evaluation of Lazarus, we also implemented very simple prototypes of a hub and a provisioning environment.

We implemented the hub in Python. The hub supports receiving AliasID certificates from the device, exchanging boot and deferral tickets and sending firmware updates. It is also able to issue an updated signed device configuration, e.g. to change Wi-Fi credentials, certificates or the hub's address.

For device provisioning, we implemented a Python script that builds (using gcc-arm-none-eabi) and flashes the different binaries. At first boot, the MCU allows access to its memory via the SWD Debug Interface using the NXP Link2 debugger to retrieve the Certificate Signing Request (CSR) for the DeviceID certificate, static_sym and dev_uuid, and to deploy the signed DeviceID along with the trust anchors for the hub and for code signing. The script also writes a device configuration data structure containing initial Wi-Fi credentials and the hub address.
Table 2: Size in bytes of our binaries.

\begin{tabular}{lccccc}
\hline Layer & DICE++ & LZ Core & $C P$ & UD & Untrusted \\
\hline Size (bytes) & 10,720 & 43,156 & 7,676 & 22,332 & 1,640 \\
\hline
\end{tabular}

\section{EVALUATION}

In this section, we analyze our prototype regarding its code size, its boot and runtime performance impact, as well as regarding its networking overhead. In the experiments in which the device communicates with the hub, we ran our simple hub prototype on a Lenovo Thinkpad T490s (with an Intel Core i7-8665U processor, 8 GB of DDR4 RAM and an Intel AC9560 wireless adapter) running Ubuntu 18.04 LTS and Python 3.6. The hub and the device communicated via Wi-Fi through a lightly loaded AVM Fritz!Box 7362 SL wireless router.

Code size: Table 2 shows the size of the Lazarus binaries. Untrusted is the part of Lazarus that runs as part of the untrusted business logic, mainly the fetching of boot and deferral tickets. We calculated the Untrusted size as the difference between the sizes of the business logic binaries compiled with and without Lazarus. In sum, Lazarus takes up about $93 \mathrm{kB}$, which is about $14 \%$ of the flash memory on the NXP LPC55S69. Lazarus thus leaves enough resources for business logic. Our prototype has not been subject to extensive code and binary optimization. Not depicted in Table 2, Lazarus reserves $8 \mathrm{kB}$ of RAM, i.e., 2.5\%, mapped to the secure world, unavailable to the non-secure world.

Boot time: We measured the boot time overhead as the time that elapses from the execution of the first instruction of the Lazarus boot loader to the execution of the first instruction of the business logic. We measured this time with an attached external device as our measurement tool. We used a second NXP LPC55S69 MCU. We added instructions at the start and the end of the execution of the boot loader to toggle a dedicated GPIO pin to signal the measurement device to take a timestamp. We conducted 100 separate runs. We present average values only, as the standard deviation was below $1 \%$.

The results are displayed in Table 3. In the case in which Lazarus Core boots the business logic directly, we measured a total overhead of less than 1.5 seconds. Most of this time is spent computing cryptographic primitives, e.g., boot ticket verification, in Lazarus Core using the RIoT crypto library [27] which does not use the MCU's crypto accelerators. We consider this measurement a worst-case estimate, showing that Lazarus also performs well on MCUs without cryptographic accelerators. Drastic speed-ups can be achieved using accelerators. For example, according to the documentation for the NXP LPC55S69, computing ECDSA-secp256r1 Sign + Verify takes only $116 \mathrm{~ms}$ [35].

Without a valid boot ticket, Lazarus Core runs the update downloader. In our scenario, the downloader contacts the hub with its AliasID, retrieves and stages a boot ticket and resets the device. This takes around 3.4 seconds in total.

The third part of Table 3 displays the total boot time if the hub requires a firmware update to be installed. We have omitted the individual stages of this process, as large parts of it are a repetition 
Table 3: Impact on boot time, depending on the presence of a valid boot ticket in the staging area.

\begin{tabular}{lccc}
\hline Boot Path & Layer & Time Layer $(\mathrm{ms})$ & Time Total $(\mathrm{ms})$ \\
\hline \multirow{2}{*}{ w. Ticket } & DICE++ & 152 & 152 \\
& LZ Core & 1,297 & 1,449 \\
\hline \multirow{2}{*}{ w/o Ticket } & DICE++ & 152 & 152 \\
& LZ Core & 1,025 & 1,178 \\
& UD & 754 & 1,932 \\
& DICE++ & 152 & 2,084 \\
& LZ Core & 1,310 & 3,394 \\
\hline \multirow{2}{*}{ w. patch } & & & 15,720 \\
\hline
\end{tabular}

of the second case (i.e., no boot ticket). After booting through DICE++ and Lazarus Core, the update downloader contacts the hub and receives instructions to download a firmware update. In the experiment in Table 3, it downloads and stages a $58 \mathrm{~KB}$ update containing FreeRTOS and several applications. After two more reset cycles, which install the update and obtain a boot ticket, the device finally boots into the newly installed firmware. As shown in Table 3 , the entire process takes less than 16 seconds.

Runtime overhead: We measured the runtime overhead of Lazarus against a workload consisting of FreeRTOS running our two apps: the traffic light controller and the ADC sensor app. For the baseline measurements, we ran this workload unmodified and without the Lazarus TrustZone component interposing between the workload and the hardware. For example, flash writes performed by the workload go directly to flash memory.

The Lazarus variant of the experiment runs the Lazarus TrustZone component which disables direct access by FreeRTOS and the applications to the flash storage controller, the power controller and the watchdog timer. As an alternative, Lazarus exposes the TrustZone handler functions described in Section 5. The FreeRTOS workload is recompiled with libraries that redirect accesses to the three devices to the TrustZone handlers. In addition, we run a TEETrigger service application on FreeRTOS that obtains a deferral ticket from the hub over the network and passes the ticket to TEETrigger running in TrustZone.

In each case, we used the FreeRTOS benchmarking feature to measure the CPU utilization. We compared the CPU idle time of the bare FreeRTOS app without Lazarus to our Lazarus implementation to obtain the total overhead.

Our measurements are summarized in Table 4. We measured a total overhead of $0.8 \%$ when acquiring a deferral ticket every minute with our task in FreeRTOS and verifying it with TEETrigger. A deferral ticket fetching interval of one or only a few minutes is unreasonably short and was chosen only to demonstrate that the overhead remains low even in this extreme case. Fetching deferral tickets in intervals of multiple hours or days is more realistic for most IoT use cases and leads to only negligible overhead.

In addition, we measured the overhead of only writing one flash page $(512 \mathrm{kB})$ with and without Lazarus, i.e. writing to flash via the handler versus writing to flash directly. This overhead is about $0.19 \%$ and thus negligible.
Table 4: Runtime performance impact for ticket fetching and verification with different deferral intervals.

\begin{tabular}{lccccc}
\hline Ticket Fetch Interval & $1 \mathrm{~min}$ & $5 \mathrm{~min}$ & $10 \mathrm{~min}$ & $1 \mathrm{~h}$ & $24 \mathrm{~h}$ \\
\hline Perf. Impact $(\%)$ & 0.80 & 0.16 & 0.08 & 0.01 & 0.001 \\
\hline
\end{tabular}

Table 5: Time required for ticket fetching and verification.

\begin{tabular}{lcccc}
\hline & Total & Non-Secure & Secure & Network \\
\hline Fetch Time $(\mathrm{ms})$ & 455 & 145 & 310 & 737 \\
\hline
\end{tabular}

Table 6: TCP payload sizes in bytes for different messages exchanged between device and hub.

\begin{tabular}{lccc}
\hline Message Type & Bytes Total & Request & Response \\
\hline Boot Ticket & 288 & 144 & 144 \\
Deferral Ticket & 288 & 144 & 144 \\
Send AliasID (max) & 1,003 & 1,001 & 2 \\
\hline
\end{tabular}

In a second experiment, we measured the (wall clock) time required for fetching and verifying a single deferral ticket. We used the same measurement methodology as in the boot time experiment, using an external device to take time stamps based on GPIO signals. Once again, we repeated each experiment 100 times. Table 5 shows the time spent on computing tasks (Total) and the time that elapsed between sending a ticket request to the hub and receiving the ticket (Network). The latter time does not affect the overhead, as FreeRTOS can assign the processor to other tasks in the meantime. The table also shows how the total compute time is split between the TrustZone handler (Secure) and the untrusted service application (Non-secure).

Networking: Table 6 shows the TCP payload sizes for various messages exchanged with the hub. Requesting tickets is implemented as sending and receiving a Lazarus authenticated header containing a type-field, nonce, digest and signature as well as a payload. In case of a boot ticket, the payload is just a fixed 4 byte value, in case of a deferral ticket, the payload is the requested deferral time in milliseconds, also as a 4 byte value. This header is of size 140 bytes, totaling response and reply with payload to 288 bytes. Sending the AliasID certificate to the hub takes less than one kilobyte, depending on the actual size of the certificate, which can vary.

The total TCP payload size of a boot in the update downloader path with subsequent ticket fetching in the business logic is less than 2,800 bytes, omitted from Table 6 . This includes sending the AliasID and retrieving the boot ticket in the downloader, and subsequent sending of the AliasID and retrieval of both a deferral and boot ticket in the business logic. After a normal boot with a valid boot ticket, data of less than 1,600 bytes are exchanged, i.e., sending the AliasID in the business logic and retrieving a boot and deferral ticket. Subsequent fetches of deferral tickets require only 288 bytes. 
Summary: To summarize, our evaluation shows that the overhead in terms of communication, flash and RAM requirements is modest even for resource-constrained devices. The same holds for the runtime overhead for the communication with the hub. In contrast, the increase in boot times until executing business logic is not negligible. However, we expect that resetting a device is an infrequent event, e.g., when a device needs to be serviced, or when the hub suspects misbehavior. The result is a good tradeoff between performance and security gain. We seek to improve the required boot time with a more optimized implementation in future versions, e.g., using hardware cryptographic accelerators.

\section{SECURITY DISCUSSION}

Our goal is to ensure recoverability of IoT devices even if the untrusted software is completely compromised. We achieve this goal if the hub is able to enforce the software stack the device executes within a time bound. This means that soon after the hub decides to deploy new software, i.e., an update/patch, onto the device, our recovery TCB must be executed to retrieve and apply a new version. We must assume that the attacker not only deploys malware that tries to resist recovery but also tries to permanently render devices useless. Our attacker with full control over untrusted software and possibly with access to the communication channel could try to use the following vectors to attack availability:

A-1 Manipulate or block the communication channel between device and hub.

A-2 Attempt to tamper with Lazarus by interfering with its execution, by overwriting it or by forging updates.

A-3 Tamper with peripherals to permanently render devices irrecoverable. Examples are wear out of flash storage, shutting devices down or setting them into irrecoverable low-power states, or manipulating the firmware of other peripherals.

A-4 Prevent or defer device reset, e.g, by manipulating or turning off TEETrigger or by forging deferral tickets.

A-5 Inject malware into untrusted software and try to persist it across resets.

A-6 Deceive the hub about the application of updates to Lazarus.

A-1 is only possible for a limited time. Our attack model states that attacks on the communication channel can eventually be detected and removed by the Internet Service Provider (ISP). The effect of Denial of Service (DoS) attacks on ticket fetching or hub availability were already discussed by Xu et al. [54] who observed that DoS attacks typically last less than 48 hours [43].

To protect against A-2 and A-3, we isolated the trusted components and critical peripherals from untrusted software. We used a TEE to latch trusted components and to map critical peripherals into the secure world. To enforce this, we simply configured TrustZone-M and the secure AHB controller on our prototype. Thus, an attacker is unable to misuse critical peripherals, e.g., overwriting Lazarus in flash, causing flash wear out, deactivating TEETrigger's WDT, or by switching into an irrecoverable low power state.

We kept the interface between the untrusted and the trusted components very simple. The runtime component contains only TEETrigger and the handlers for critical peripherals. TEETrigger only verifies a simple, fixed data structure, and the flash write handler, merely evaluates whether flash writes are excessive or target a certain area. The runtime component does not process any secrets. Thus, our system is by design not susceptible to sidechannel attacks. Untrusted input to Lazarus Core processed during boot time must be located in the staging area. Every structure in the staging area has a well-defined format and requires a valid signature from the hub. For the verification of all data structures, we provisioned the device with public keys in a secure environment.

We prevent A- 4 by placing TEETrigger inside the TEE and by using a simple interface into the TEE. This ensures the correct execution of TEETrigger which, by construction, will force a device reset unless regularly provided with a new deferral ticket from the hub. Deferral tickets are freshness-protected and only valid when signed with the hub private key.

Regarding A-5, the attacker can compromise the untrusted software according to our threat model. The attacker's code can run on the device until the next reset. The hub can force such a reset by refusing to issue TEETrigger deferral tickets (e.g., because it became aware of the attack).

If the attack was not persistent, the reset will remove it from the device. The attacker may be able to reinfect the device using the same method as the first time (e.g., exploiting the same vulnerability). Either way, the hub can (and should) deploy a software update that disables future attacks (e.g., by patching the vulnerability). Lazarus enables the hub to do so irrespective of the state of the device.

If the attack persisted itself, it must have changed storage. If this change affected storage that is hashed by DICE (e.g., executable code), the hash values will also change. Thus, attestation to the hub will fail, and the hub can force a reset and update as in the nonpersistent case.

One can also envision data-only vulnerabilities that allow an attacker to take control by changing data the hashed code will read (e.g., configuration data read during OS boot) but that are not included in the hash because they can change over time. While such persistent changes would not manifest themselves in changed DICE hashes, the device could still be recovered unconditionally by using Lazarus to patch the vulnerable code and the exploit data.

If the downloader is free of flaws, it can retrieve updates without obstacle. However, we did not assume that the downloader is free of vulnerabilities. Lazarus latches the downloader in flash, such that malware cannot overwrite it. It also activates TEETrigger before running the downloader. This ensures that a reset will eventually occur. A DoS on the downloader is possible as long as it can be re-compromised after each reset. The hub can detect such attacks (as it will fail to see device attestations for the software update) and block the attack with the help of the ISP.

For defending against A-6, we pointed out non-forgeability and freshness of the relevant cryptographic messages sent between the device and the hub in Subsection 4.4. This only allows devices that indeed applied an update of a trusted component to provide proof to the hub.

In summary, Lazarus allows a device to be reliably recovered and its software (including Lazarus) to be updated within a time bound that can be configured by the hub. 


\section{APPLICATION IN REAL-WORLD USE CASES}

Before applying Lazarus in a real-world use case, the developer has to configure Lazarus based on the peripherals on the device and based on the demands of the target application. This may require writing new trusted handlers to regulate access to critical peripherals and tailoring our prototype to concrete IoT scenarios, device types and specific peripherals, e.g., sensors or additional flash storage attached via Serial Peripheral Interface (SPI). In particular, all peripherals that can possibly be misused by compromised business logic have to be guarded by TEE handlers. For example, flash write handlers must implement a threshold or rate limiting to distinguish regular write activities from targeted flash wear out. In addition, business logic must be implemented such that it does not exceed handler thresholds during regular operation. This should be easy since the behavior of many IoT applications is quite regular and known in advance.

In addition to writing trusted handlers, the developer has to modify the IoT business logic to call these handlers instead of trying to access the peripherals directly. Such attempts would fail, as the peripherals are protected by TrustZone from access by untrusted software.

Further, integration of Lazarus requires setting a reasonable deferral timeout and determining the desired behavior in case the device cannot contact the hub, e.g. because of a DoS attack, as discussed in [54]. A possibility is to boot into a safe-mode version of the software with minimal functionality as long as the hub is unavailable. However, this strongly depends on the IoT use case the system is tailored for.

Lazarus can accommodate use cases that include code running in a TEE by running both the Lazarus runtime component and the business logic's TEE code in the TEE. Depending on the size and the quality of the latter, this may increase the attack surface of the TEE code.

\section{RELATED WORK}

Mechanisms for remote administration of servers have been established in several industry standards [11, 21, 22]. These standards enable the efficient remote configuration, monitoring and update of a large number of devices irrespective of the state of their application processor and running software. Devices are equipped with an additional co-processor able to interrupt the application processor, and possibly with a separate physical network interface. This allows administrators to restore hung or compromised systems, or even to activate servers that are shut down. This achieves recoverability. However, the system design of adding isolated co-processors and possibly separate network interfaces is not an option for IoT devices in the low-cost segment, especially not for constrained devices.

Several mechanisms for automated malware detection and eviction from end-user devices have been proposed [15, 19, 28]. This line of work focuses on security in a higher layer and has a large TCB, i.e., the OS kernel, a component Lazarus considers untrusted. An architecture which leverages system virtualization for automated detection and containment of rootkit attacks on Linux systems was proposed by Baliga et al. [6]. Lazarus also considers hypervisors untrusted components and can recover them.
Android Things [16] is a commercial secure IoT platform. It can be regarded as a full-fledged trustworthy IoT ecosystem with an OS and a bootloader for compliant device architectures which enforces secure boot, runtime isolation, attestation, hardware-based key protection, and handles remote updates. IoT use cases are engineered on top of the platform and can make use of the security services the OS provides, e.g., for attestation or authentication. The focus is on powerful Cortex-A devices. There is, to the best of our knowledge, no means of recovering from a compromised OS.

For constrained devices, several secure update mechanisms have been proposed, mainly for sensor networks in combination with attestation $[1,14,20,23,36,40,41]$. After trying to distribute the update, the server requests an attestation proof of successful update application. If the update fails (e.g., because malware is preventing it), the compromised devices are revoked and require manual repair. In contrast, Lazarus permits remote recovery of all devices. Some mechanisms allow wiping malware, e.g., with secure memory erasure $[1,20,36]$. However, common to all mechanisms is that they cannot reliably recover from compromise of the host system and that they have no provision to force execution of recovery functionality.

Asokan et al. [4] propose an architecture for secure software updates on MCUs. They keep the secure update mechanism and key material in read-only segments protected from the rest of the possibly malicious system. They built prototypes on two device types, HYDRA and Cortex-M. The update functionality on HYDRA is loaded as the initial user space process with highest priority after starting an seL4 kernel upon successful secure boot. The isolation of the task's code and data is ensured by the seL4 kernel's separation. In contrast, our goal is to decouple the recovery TCB from OSs or hypervisors. The Cortex-M prototype uses TrustZone-M for isolation and executes the update functionality as part of a trusted bootloader. Asokan et al. do not propose a mechanism that returns control to the recovery TCB after infection and provide no way to service the read-only update functionality.

CIDER [54] is most closely related to our work and lays the architectural foundation for Lazarus. CIDER appears to be targeted primarily at higher-end devices for which security features such as storage latches are common. The higher baseline cost of such devices $(\geq \$ 100)$ also makes it reasonable to add missing security support by attaching hardware costing a few dollars to an extension port. CIDER did this for its external AWDT. The situation is fundamentally different for the cheap low-end boards which are the focus of our work. While CIDER was also implemented on a low-end MCU, this prototype inherits the properties of its higherend cousins, including an AWDT implementation on a separate MCU board. The paper mentions cheaper alternatives without exploring them. In contrast, Lazarus demonstrates how a wide range of protections can be implemented at zero cost in TEE software. In addition, Lazarus provides protection against a range of attacks aiming to disable the device and enables easy updates of the entire TCB.

The TCG resilience work group provides no concrete implementation [52]. Auer et al. [5] only mention integration of an AWDT and recovery TCB into a secure architecture for RISC-V. 


\section{CONCLUSION}

We present Lazarus, a system for the recovery of compromised low-end IoT devices. Lazarus overcomes three major challenges in the design of cyber-resilient IoT architectures. These challenges are applicability on low-cost COTS devices, defense against malware actively trying to make devices unavailable, and practical updates of the recovery TCB. Lazarus uses a TEE to constrain malware.

As TEEs are nowadays available even on low-cost devices, Lazarus can be deployed on a broad range of COTS devices. We use the TEE to latch the recovery TCB, isolate our reset trigger TEETrigger and regulate access to critical peripherals. The latter prevents malware from rendering devices permanently unavailable through misuse of peripherals, such as entering irrecoverable sleep states or flash wear out, otherwise possible within minutes [46]. For practical updates of the recovery TCB, we propose an extension to DICE. This extension allows sustaining the device identity through secure re-association with the hub.

We have implemented Lazarus on a COTS ARMv8-M MCU featuring TrustZone-M. Our prototype has low memory requirements, negligible runtime performance impact and modest boot time impact, making Lazarus suitable for adoption in a broad range of IoT use cases.

\section{REFERENCES}

[1] Mahmoud Ammar, Wilfried Daniels, Bruno Crispo, and Danny Hughes. 2018 SPEED: Secure Provable Erasure for Class-1 IoT Devices. In Proceedings of the Eighth ACM Conference on Data and Application Security and Privacy (Tempe, AZ, USA) (CODASPY '18). ACM, New York, NY, USA, 111-118. https://doi.org/ $10.1145 / 3176258.3176337$

[2] ARM. 2019. AMBA 5 Overview. https://developer.arm.com/architectures/systemarchitectures/amba/amba-5.

[3] ARM Limited. 2017. Introduction to the ARMv8-M architecture.

[4] N. Asokan, T. Nyman, N. Rattanavipanon, A.-R. Sadeghi, and G. Tsudik. 2018. AS SURED: Architecture for Secure Software Update of Realistic Embedded Devices. ArXiv e-prints (July 2018). arXiv:1807.05002 [cs.CR]

[5] L. Auer, C. Skubich, and M. Hiller. 2019. A Security Architecture for RISC-V based IoT Devices. In 2019 Design, Automation Test in Europe Conference Exhibition (DATE). 1154-1159. https://doi.org/10.23919/DATE.2019.8714822

[6] Arati Baliga, Xiaoxin Chen, and Liviu Iftode. 2006. Paladin: Automated detection and containment of rootkit attacks. Department of Computer Science, Rutgers University (2006).

[7] Katharina Bogad and Manuel Huber. 2019. Harzer Roller: Linker-Based Instrumentation for Enhanced Embedded Security Testing. In Proceedings of the 3rd Reversing and Offensive-Oriented Trends Symposium (Vienna, Austria) (ROOTS'19). Association for Computing Machinery, New York, NY, USA, Article 3, 9 pages. https://doi.org/10.1145/3375894.3375897

[8] Credencys. 2019. Increase your Vending Machine's performance efficiency while bringing down the operational expenses \& maintenance cost with IoT. https: //www.credencys.com/smart-vending-machine-iot-solutions/.

[9] Daimler. 2019. Networked with the surroundings. Car-to-X communication goes into series production. https:/www.daimler.com/innovation/case/connectivity/ car-to-x-2.html.

[10] J. Deogirikar and A. Vidhate. 2017. Security attacks in IoT: A survey. In 2017 International Conference on I-SMAC (IoT in Social, Mobile, Analytics and Cloud) (I-SMAC). 32-37. https://doi.org/10.1109/I-SMAC.2017.8058363

[11] Distributed Management Task Force. 2018. Redfish Scalable Platforms Management API Specification v1.5.

[12] DZone. 2019. Home Automation Using IoT. https://dzone.com/articles/homeautomation-using-iot.

[13] Espressif Systems. 2019. ESP8266EX Datasheet.

[14] Wei Feng, Yu Qin, Shijun Zhao, Ziwen Liu, Xiaobo Chu, and Dengguo Feng. 2017. Secure Code Updates for Smart Embedded Devices based on PUFs. Cryptology ePrint Archive, Report 2017/991. https://eprint.iacr.org/2017/991.

[15] J. Giffin. 2010. The Next Malware Battleground: Recovery After Unknown Infection. IEEE Security Privacy 8, 3 (May 2010), 74-76. https://doi.org/10.1109/MSP 2010.107

[16] Google. 2018. Android Developers: Android Things. https://developer.android. com/things/.
[17] HardwareBee. 2019. MCU Market History and Forecast 2016-2023. http: //hardwarebee.com/mcu-market-history-and-forecast-2016-2023/.

[18] Hilti. 2019. White paper: Introducing Digital Asset Management. https://www.hilti.com/content/dam/documents/pdf/w1/ontrack/whitepapers/ W1_US_en_White\%20Paper\%20Increase\%20Profit\%20and\%20Productivity.pdf.

[19] F. Hsu, H. Chen, T. Ristenpart, J. Li, and Z. Su. 2006. Back to the Future: A Framework for Automatic Malware Removal and System Repair. In 2006 22nd Annual Computer Security Applications Conference (ACSAC'06). 257-268. https: //doi.org/10.1109/ACSAC.2006.16

[20] C. Huth, P. Duplys, and T. Güneysu. 2016. Secure Software Update and IP Protection for Untrusted Devices in the Internet of Things via Physically Unclonable Functions. In 2016 IEEE International Conference on Pervasive Computing and Communication Workshops (PerCom Workshops). 1-6. https://doi.org/10.1109/ PERCOMW.2016.7457156

[21] Intel Corporation. 2011. Data Center Manageability Interface Specification v1.5 rev. 1.0 .

[22] Intel Corporation. 2013. Intelligent Platform Management Interface Specification v2.0 rev. 1.1.

[23] Florian Kohnhäuser and Stefan Katzenbeisser. 2016. Secure Code Updates for Mesh Networked Commodity Low-End Embedded Devices. In Computer Security - ESORICS 2016, Ioannis Askoxylakis, Sotiris Ioannidis, Sokratis Katsikas, and Catherine Meadows (Eds.). Springer International Publishing, Cham, 320-338.

[24] Ben Lapid and Avishai Wool. 2018. Cache-Attacks on the ARM TrustZone implementations of AES-256 and AES-256-GCM via GPU-based analysis. Cryptology ePrint Archive, Report 2018/621. https://eprint.iacr.org/2018/621.

[25] OpenSystems Media. 2019. IoT Based Smart Traffic Signal Monitoring Using Vehicle Count. https://www.embedded-computing.com/guest-blogs/iot-basedsmart-traffic-signal-monitoring-using-vehicle-count.

[26] Microchip. 2020. CEC1702. https://www.microchip.com/wwwproducts/en/ CEC1702.

[27] Microsoft Research. 2019. Robust Internet of Things. https://github.com/ microsoft/RIoT.

[28] Yacin Nadji, Jonathon Giffin, and Patrick Traynor. 2011. Automated Remote Repair for Mobile Malware. In Proceedings of the 27th Annual Computer Security Applications Conference (Orlando, Florida, USA) (ACSAC '11). ACM, New York, NY, USA, 413-422. https://doi.org/10.1145/2076732.2076791

[29] M. Nawir, A. Amir, N. Yaakob, and O. B. Lynn. 2016. Internet of Things (IoT): Taxonomy of security attacks. In 20163 rd International Conference on Electronic Design (ICED). 321-326. https://doi.org/10.1109/ICED.2016.7804660

[30] NetScout. 2019. Mirai IoT Botnet Description and DDoS Attack Mitigation. https://www.netscout.com/blog/asert/mirai-iot-botnet-description-andddos-attack-mitigation.

[31] NXP. 2019. AN12278 - LPC55S69 Security Solutions for IoT.

[32] NXP. 2019. LPC55S69-EVK: LPCXpresso55S69 Development Board. https://www.nxp.com/products/processors-and-microcontrollers/armmicrocontrollers/general-purpose-mcus/lpc5500-cortex-m33/lpcxpresso55s69development-board:LPC55S69-EVK.

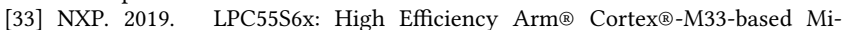
crocontroller Family. https://www.nxp.com/products/processors-andmicrocontrollers/arm-microcontrollers/general-purpose-mcus/lpc5500-cortexm33/high-efficiency-arm-cortex-m33-based-microcontroller-family:LPC55S6x.

[34] NXP. 2019. LPC55Sxx Secure Boot. https://www.nxp.com/docs/en/applicationnote/AN12283.pdf.

[35] NXP. 2019. UM11126 LPC55S6x/LPC55S2x/LPC552x User manual.

[36] Daniele Perito and Gene Tsudik. 2010. Secure Code Update for Embedded Devices via Proofs of Secure Erasure. In Proceedings of the 15th European Conference on Research in Computer Security (Athens, Greece) (ESORICS'10). Springer-Verlag, Berlin, Heidelberg, 643-662. http://dl.acm.org/citation.cfm?id=1888881.1888931

[37] Cauligi S Raghavendra, Krishna M Sivalingam, and Taieb Znati. 2006. Wireless sensor networks. Springer.

[38] Arjmand Sanuel. 2017. Azure IoT supports new security hardware to strengthen IoT security. https:/azure.microsoft.com/en-us/blog/azure-iot-supports-newsecurity-hardware-to-strengthen-iot-security/.

[39] Amazon Web Services. 2019. freeRTOS. https://www.freertos.org/.

[40] Arvind Seshadri, Mark Luk, Adrian Perrig, Leendert van Doorn, and Pradeep Khosla. 2004. Using FIRE \& ICE for Detecting and Recovering Compromised Nodes in Sensor Networks.

[41] Arvind Seshadri, Mark Luk, Adrian Perrig, Leendert van Doorn, and Pradeep Khosla. 2006. SCUBA: Secure Code Update By Attestation in Sensor Networks. In Proceedings of the 5th ACM Workshop on Wireless Security (Los Angeles, California) (WiSe '06). ACM, New York, NY, USA, 85-94. https://doi.org/10.1145/1161289. 1161306

[42] Sigfox. 2019. Smart livestock collars let ranchers track, monitor and manage herds like never before. https://www.sigfox.com/en/solutions/smart-livestockcollars-let-ranchers-track-monitor-and-manage-herds-never.

[43] Symantec. 2016. Internet Security Threat Report.

[44] Symantec. 2019. Hajime worm battles Mirai for control of the Internet of Things. https://www.symantec.com/connect/blogs/hajime-worm-battles-mirai- 
control-internet-things.

[45] TC Group and others. 2011. TPM Main Specification Version 1.2 Rev. 116. https //trustedcomputinggroup.org/resource/tpm-main-specification/.

[46] R. Templeman and A. Kapadi. 2012. GANGRENE: Exploring the Mortality of Flash Memory. In Presented as part of the 7th USENIX Workshop on Hot Topics in Security USENIX, Bellevue, WA. https://www.usenix.org/conference/hotsec12/workshopprogram/presentation/Templeman

[47] Terry Yin. 2019. A simple code complexity analyzer without caring about the $\mathrm{C} / \mathrm{C}++$ header files or Java imports. https://terryyin.github.io/lizard/.

[48] Ankit Tiwari, Prasanna Ballal, and Frank L. Lewis. 2007. Energy-efficient Wireless Sensor Network Design and Implementation for Condition-based Maintenance. ACM Trans. Sen. Netw. 3, 1, Article 1 (March 2007). https://doi.org/10.1145/ 1210669.1210670

[49] Trusted Computing Group. 2017. Foundational Trust for IoT and Resource Constrained Devices.
[50] Trusted Computing Group. 2018. Hardware Requirements for a Device Identifier Composition Engine.

[51] Trusted Computing Group. 2018. Implicit Identity Based Device Attestation.

[52] Trusted Computing Group. 2019. TCG Cyber Resilient Technologies. https://trustedcomputinggroup.org/wp-content/uploads/TCG-Cyber-ResilientTechnologies-\%E2\%80\%93-Rob-Spiger-Microsoft.pdf.

[53] R. Williams, E. McMahon, S. Samtani, M. Patton, and H. Chen. 2017. Identifying vulnerabilities of consumer Internet of Things (IoT) devices: A scalable approach. In 2017 IEEE International Conference on Intelligence and Security Informatics (ISI). 179-181. https://doi.org/10.1109/ISI.2017.8004904

[54] Meng Xu, Manuel Huber, Zhichuang Sun, Paul England, Marcus Peinado, Sangho Lee, Andrey Marochko, Dennis Mattoon, Rob Spiger, and Stefan Thom. 2019. Dominance as a New Trusted Computing Primitive for the Internet of Things. In Proceedings of the 2019 IEEE Symposium on Security and Privacy (San Francisco, CA) (SP '19). IEEE Computer Society, Washington, DC, USA. 